\title{
Fractional-order Riccati differential equation: Analytical approximation and numerical results
}

\author{
Najeeb Alam Khan ${ }^{1 *}$, Asmat Ara² and Nadeem Alam Khan
}

\section{"Correspondence:}

njbalam@yahoo.com

'Department of Mathematical

Sciences, University of Karachi,

Karachi, 75270, Pakistan

Full list of author information is

available at the end of the article

\section{Springer}

\begin{abstract}
The aim of this article is to introduce the Laplace-Adomian-Padé method (LAPM) to the Riccati differential equation of fractional order. This method presents accurate and reliable results and has a great perfection in the Adomian decomposition method (ADM) truncated series solution which diverges promptly as the applicable domain increases. The approximate solutions are obtained in a broad range of the problem domain and are compared with the generalized Euler method (GEM). The comparison shows a precise agreement between the results, the applicable one of which needs fewer computations.
\end{abstract}

Keywords: Adomian decomposition method (ADM); Mittag-Leffler function; Padé approximation; Riccati equation

\section{Introduction}

In recent years, it has turned out that many phenomena in biology, chemistry, acoustics, control theory, psychology and other areas of science can be fruitfully modeled by the use of fractional-order derivatives. That is because of the fact that a reasonable modeling of a physical phenomenon having dependence not only on the time instant but also on the prior time history can be successfully achieved by using fractional calculus [1]. Fractional differential equations (FDEs) have been used as a kind of model to describe several physical phenomena [2-6] such as damping laws, rheology, diffusion processes, and so on. Moreover, some researchers have shown the advantageous use of the fractional calculus in the modeling and control of many dynamical systems. Besides modeling, finding accurate and proficient methods for solving FDEs has been an active research undertaking. Exact solutions for the majority of FDEs cannot be found easily, thus analytical and numerical methods must be used. Some numerical methods for solving FDEs have been presented and they have their own advantages and limitations.

Many physical problems are governed by fractional differential equations (FDEs), and finding the solution of these equations have been the subject of many investigations in recent years. Recently, there have been a number of schemes devoted to the solution of fractional differential equations. These schemes can be broadly classified into two classes, numerical and analytical. The Adomian decomposition method [7], homotopy perturbation method [8-11], homotopy analysis method [12,13], Taylor matrix method [14] and 
Haar wavelet method [15] have been used to solve the fractional-order Riccati differential equation. However, the convergence region of the corresponding results is rather small.

In this work, the nonlinear fractional-order Riccati differential equations will be approached analytically by combining the Laplace transform, the Adomian decomposition method (ADM), and the Padé approximation. The Laplace-Adomian-Padé approximation was proposed by Tsai and Chen [16] for solving Ricatti differential equations. The method was extended by Zeng et al. [17] to derive the analytical approximate solutions of fractional differential equations. Khan et al. [18] applied the Laplace transformation coupled with the decomposition method in fractional order seepage flow and telegraph equations. We applied the idea of refs. $[16,17]$ for solving a fractional-order Riccati differential equation. The Laplace-Adomian-Padé method (LAPM) is illustrated by applications, and the results obtained are compared with those of the exact and numerical solutions by the generalized Euler method. Odibat and Momani [19] derived the generalized Euler method that was developed for the numerical solution of initial value problems with Caputo derivatives.

\section{Definitions and preliminaries}

\section{Caputo's fractional derivative}

Caputo's fractional derivative of a function $f(t)$ is defined by

$$
\frac{d^{\alpha} f}{d t^{\alpha}}=\frac{1}{\Gamma(n-\alpha)} \int_{0}^{t}(t-\tau)^{n-\alpha-1} f^{(n)}(t) d t \quad(n-1<\alpha<n) .
$$

The Laplace transform to Caputo's fractional derivative gives

$$
\mathcal{L}\left\{\frac{d^{\alpha}}{d t^{\alpha}} f(t)\right\}=s^{\alpha} F(s)-\sum_{m=0}^{n-1} s^{\alpha-m-1} f^{(m)}(0) \quad(n-1<\alpha<n) .
$$

The Mittag-Leffler function and its generalized forms have played a special role in solving the fractional differential equations. The so-called Mittag-Leffler function with two parameters $E_{\alpha, \beta}(Z)$ was introduced by Agarwal [20]

$$
E_{\alpha, \beta}(Z)=\sum_{j=0}^{\infty} \frac{Z^{j}}{\Gamma(\alpha j+\beta)} \quad(\alpha>0, \beta>0) .
$$

Its $k$ th derivative is given by [20]

$$
E_{\alpha, \beta}^{(k)}(Z)=\sum_{j=0}^{\infty} \frac{(j+k) ! Z^{j}}{j ! \Gamma(\alpha j+\alpha k+\beta)} \quad(k=0,1,2,3, \ldots) .
$$

We find it convenient to introduce the function

$$
\varepsilon_{k}(t, a: \alpha, \beta)=t^{\alpha k+\beta-1} E_{\alpha, \beta}^{(k)}\left( \pm a t^{\alpha}\right) .
$$

Its Laplace transform was evaluated by Podlubny [4]

$$
\int_{0}^{\infty} e^{-s t} \varepsilon_{k}(t, a: \alpha, \beta) d t=\frac{k ! s^{\alpha-\beta}}{\left(s^{\alpha} \mp a\right)^{k+1}} \quad\left(\operatorname{Re}(s) \succ|a|^{1 / \alpha}\right) .
$$


Hence

$$
\mathcal{L}^{-1}\left[\frac{k ! s^{\alpha-\beta}}{\left(s^{\alpha} \mp a\right)^{k+1}}\right]=t^{\alpha k+\beta-1} E_{\alpha, \beta}^{(k)}\left( \pm a t^{\alpha}\right) .
$$

Another convenient property of $\varepsilon_{k}(t, y: \alpha, \beta)$, which has been used in this paper, is its simple fractional differentiation

$$
{ }_{0} D_{t}^{\lambda} \varepsilon_{k}(t, a: \alpha, \beta)=\varepsilon_{k}(t, a: \alpha, \beta-\lambda) \quad(\lambda \prec \beta) .
$$

\section{Implementation of LAPM}

Consider the fractional-order Riccati differential equation of the form

$$
D_{t}^{\alpha} y=P(t) y^{2}+Q(t) y+R(t), \quad t>0,0<\alpha \leq 1
$$

subject to the initial condition

$$
y(0)=k_{1} \text {. }
$$

The nonlinear term in Eq. (9) is $y^{2}$ and $P(t), Q(t)$ and $R(t)$ are known functions. For $\alpha=1$, the fractional-order Riccati equation converts into the classical Riccati differential equation. Applying the Laplace transform on both sides of Eq. (9),

$$
\mathcal{L}\left[D_{t}^{\alpha} y\right]=\mathcal{L}\left[P(t) y^{2}\right]+\mathcal{L}[Q(t) y]+\mathcal{L}[R(t)]
$$

Using the property of the Laplace transform, we get

$$
s^{\alpha} \mathcal{L}[y]-s^{\alpha-1} y(0)=\mathcal{L}\left[P(t) y^{2}\right]+\mathcal{L}[Q(t) y]+\mathcal{L}[R(t)]
$$

Using the initial condition from Eq. (10), the outcome is

$$
s^{\alpha} \mathcal{L}[y]-s^{\alpha-1} k_{1}=\mathcal{L}\left[P(t) y^{2}\right]+\mathcal{L}[Q(t) y]+\mathcal{L}[R(t)] .
$$

Equation (13) can be written as

$$
\mathcal{L}[y]=\frac{k_{1}}{s}+\frac{1}{s^{\alpha}} \mathcal{L}[R(t)]+\frac{1}{s^{\alpha}} \mathcal{L}[Q(t) y]+\frac{1}{s^{\alpha}} \mathcal{L}\left[P(t) y^{2}\right] .
$$

The method assumes the solution as an infinite series:

$$
y=\sum_{n=0}^{\infty} y_{n} .
$$

The nonlinearity $y^{2}$ is decomposed as

$$
y^{2}=\sum_{n=0}^{\infty} A_{n}
$$


where $A_{n}=A_{n}\left(y_{0}, y_{1}, y_{2}, y_{3}, \ldots, y_{n}\right)$ are the so-called Adomian polynomials given as

$$
\left[\begin{array}{c}
A_{0} \\
A_{1} \\
A_{2} \\
\ldots \\
\ldots
\end{array}\right]=\left[\begin{array}{ccccc}
1 & 0 & 0 & \ldots & 0 \\
0 & y_{1} & 0 & \ldots & 0 \\
0 & y_{2} & \frac{1}{2 !} y_{1}^{2} & \ldots & 0 \\
\ldots & \ldots & \ldots & \ldots & \ldots \\
\ldots & \ldots & \ldots & \ldots & \ldots
\end{array}\right]\left[\begin{array}{c}
f\left(y_{0}\right) \\
f^{\prime}\left(y_{0}\right) \\
f^{\prime \prime}\left(y_{0}\right) \\
\ldots \\
\ldots
\end{array}\right] .
$$

Substituting Eqs. (15) and (17) into Eq. (14), the result is

$$
\mathcal{L}\left[\sum_{n=0}^{\infty} y_{n}\right]=\frac{k_{1}}{s}+\frac{1}{s^{\alpha}} \mathcal{L}\left[P(t) \sum_{n=0}^{\infty} A_{n}\right]+\frac{1}{s^{\alpha}} \mathcal{L}\left[Q(t) \sum_{n=0}^{\infty} y_{n}\right]+\frac{1}{s^{\alpha}} \mathcal{L}[R(t)] .
$$

Matching both sides of Eq. (18) yields the following iterative algorithm:

$$
\begin{aligned}
& \mathcal{L}\left[y_{0}\right]=\frac{k_{1}}{s}+\frac{1}{s^{\alpha}} \mathcal{L}[R(t)] \\
& \mathcal{L}\left[y_{1}\right]=\frac{1}{s^{\alpha}} \mathcal{L}\left[P(t) A_{0}\right]+\frac{1}{s^{\alpha}} \mathcal{L}\left[Q(t) y_{0}\right], \\
& \mathcal{L}\left[y_{2}\right]=\frac{1}{s^{\alpha}} \mathcal{L}\left[P(t) A_{1}\right]+\frac{1}{s^{\alpha}} \mathcal{L}\left[Q(t) y_{1}\right], \\
& \vdots \\
& \mathcal{L}\left[y_{n}\right]=\frac{1}{s^{\alpha}} \mathcal{L}\left[P(t) A_{n-1}\right]+\frac{1}{s^{\alpha}} \mathcal{L}\left[Q(t) y_{n-1}\right]
\end{aligned}
$$

The aim is to study the mathematical behavior of the solution $y(t)$ for different values of $\alpha$. By applying the inverse Laplace transform to both sides of Eq. (19), the value of $y_{0}$ is obtained. Substituting these values of $y_{0}$ and $A_{0}$ into Eq. (20), the first component $y_{1}$ is obtained. The other terms $y_{2}, y_{3}, y_{4}, \ldots$ can be calculated recursively in a similar way by Eqs. (20)-(22). The LAPM solution coincides with the Taylor series solution in the initial value case and diverges rapidly as the applicable domain increases. This goal can be achieved by forming Padé approximants, which have the advantage of manipulating the polynomial approximation into a rational function to gain more information about $y(t)$. It is well known that Padé approximants will converge on the entire real axis, if $y(t)$ is free of singularities on the real axis. To consider the behaviors of a solution for different values of $\alpha$, we will take advantage of Eq. (15) available for $0<\alpha \leq 1$.

\section{Test problems}

In this section, we implement LAPM to the nonlinear fractional Riccati differential equations. Two examples of nonlinear fractional Riccati differential equations are solved with real coefficients.

Test problem 1. Consider the nonlinear Riccati differential equation

$$
D_{t}^{\alpha}(y)=1+2 y(t)-y^{2}(t), \quad 0<\alpha \leq 1,
$$

with the initial condition

$$
y(0)=0 \text {. }
$$


The exact solution for $\alpha=1$ was found to be

$$
y(t)=1+\sqrt{2} \tanh \left[\sqrt{2} t+\frac{1}{2} \log \left(\frac{\sqrt{2}-1}{\sqrt{2}+1}\right)\right] .
$$

First, applying the Laplace transform on both sides of Eq. (23), we get

$$
\mathcal{L}\left[D_{t}^{\alpha}(y)\right]=\mathcal{L}[1]+2 \mathcal{L}[y]-\mathcal{L}\left[y^{2}\right]
$$

Using the property of the Laplace transform, we obtain

$$
s^{\alpha} \mathcal{L}[y]-s^{\alpha-1} y(0)=\frac{1}{s}+2 \mathcal{L}[y]-\mathcal{L}\left[y^{2}\right] .
$$

Using the initial condition from Eq. (24), it becomes

$$
\mathcal{L}[y]=\frac{1}{s\left(s^{\alpha}-2\right)}-\frac{1}{s^{\alpha}-2} \mathcal{L}\left[y^{2}\right]
$$

Substituting Eqs. (15) and (16) into Eq. (28), the result is

$$
\mathcal{L}\left[\sum_{n=0}^{\infty} y_{n}\right]=\frac{1}{s\left(s^{\alpha}-2\right)}-\frac{1}{s^{\alpha}-2} \mathcal{L}\left[P(t) \sum_{n=0}^{\infty} A_{n}\right]
$$

Matching both sides of Eq. (29) yields

$$
\begin{aligned}
& \mathcal{L}\left[y_{0}\right]=\frac{1}{s\left(s^{\alpha}-2\right)}, \\
& \mathcal{L}\left[y_{1}\right]=-\frac{1}{s^{\alpha}-2} \mathcal{L}\left[A_{0}\right], \\
& \mathcal{L}\left[y_{2}\right]=-\frac{1}{s^{\alpha}-2} \mathcal{L}\left[A_{1}\right], \\
& \vdots \\
& \mathcal{L}\left[y_{n}\right]=-\frac{1}{s^{\alpha}-2} \mathcal{L}\left[A_{n-1}\right] \quad(n \geq 1) .
\end{aligned}
$$

Applying the inverse fractional Laplace transform to Eq. (30), hence we can write it as

$$
\mathcal{L}\left[y_{0}\right]=\frac{s^{-1}}{s^{\alpha}-2}
$$

By applying the inverse Laplace transform to Eq. (34), the value $y_{0}$ is obtained as

$$
y_{0}=t^{\alpha} E_{\alpha, 1+\alpha}\left(2 t^{\alpha}\right)=t^{\alpha} \sum_{j=0}^{\infty} \frac{\left(2 t^{\alpha}\right)^{j}}{\Gamma((j+1) \alpha+1)} .
$$

Now, considering the few terms of $y_{0}$,

$$
y_{0}=t^{\alpha}\left(\frac{1}{\Gamma(\alpha+1)}+\frac{\left(2 t^{\alpha}\right)}{\Gamma(2 \alpha+1)}+\frac{\left(2 t^{\alpha}\right)^{2}}{\Gamma(3 \alpha+1)}+\frac{\left(2 t^{\alpha}\right)^{3}}{\Gamma(4 \alpha+1)}+\frac{\left(2 t^{\alpha}\right)^{5}}{\Gamma(5 \alpha+1)}+\cdots\right) \text {. }
$$


The first Adomian polynomial $A_{0}$ is obtained from Eq. (17), then we substitute $y_{0}$ and $A_{0}$ in Eq. (31). Evaluating the Laplace transform of the quantities on the right-hand side of Eq. (31) and then applying the inverse Laplace transform, the value of $y_{1}$ can be obtained. The other terms $y_{2}, y_{3}, \ldots$ can be computed recursively in a similar calculation. By using LAPM, a power series solution is essentially a truncated series solution. The LAPM solution coincides with the Maclaurin series of the exact solution in the initial value case and diverges rapidly as the applicable domain increases. The next two components of the solution are

$$
\begin{aligned}
y_{1}= & -\frac{t^{3 \alpha} \Gamma(1+2 \alpha)}{(\Gamma(1+\alpha))^{2} \Gamma(1+3 \alpha)}-\frac{2 t^{4 \alpha} \Gamma(1+2 \alpha)}{(\Gamma(1+\alpha))^{2} \Gamma(1+4 \alpha)}-\frac{4 t^{4 \alpha} \Gamma(1+3 \alpha)}{\Gamma(1+\alpha) \Gamma(1+2 \alpha) \Gamma(1+4 \alpha)} \\
& -\frac{4 t^{5 \alpha} \Gamma(1+2 \alpha)}{(\Gamma(1+\alpha))^{2} \Gamma(1+5 \alpha)}-\frac{8 t^{5 \alpha} \Gamma(1+3 \alpha)}{\Gamma(1+\alpha) \Gamma(1+2 \alpha) \Gamma(1+5 \alpha)} \\
& -\frac{4 t^{5 \alpha} \Gamma(1+4 \alpha)}{(\Gamma(1+2 \alpha))^{2} \Gamma(1+5 \alpha)}-\cdots, \\
y_{2}= & \frac{2 t^{5 \alpha} \Gamma(1+2 \alpha) \Gamma(1+4 \alpha)}{\Gamma(1+\alpha)^{3} \Gamma(1+3 \alpha) \Gamma(1+5 \alpha)}+\frac{4 t^{6 \alpha} \Gamma(1+2 \alpha) \Gamma(1+4 \alpha)}{\Gamma(1+\alpha)^{3} \Gamma(1+3 \alpha) \Gamma(1+6 \alpha)} \\
& +\frac{4 t^{6 \alpha} \Gamma(1+5 \alpha)}{\Gamma(1+\alpha)^{3} \Gamma(1+3 \alpha) \Gamma(1+6 \alpha)}+\frac{4 t^{6 \alpha} \Gamma(1+2 \alpha) \Gamma(1+5 \alpha)}{\Gamma(1+\alpha)^{3} \Gamma(1+4 \alpha) \Gamma(1+6 \alpha)} \\
& +\frac{8 t^{6 \alpha} \Gamma(1+3 \alpha) \Gamma(1+5 \alpha)}{\Gamma(1+\alpha)^{2} \Gamma(1+2 \alpha) \Gamma(1+4 \alpha) \Gamma(1+6 \alpha)}+\cdots
\end{aligned}
$$

Therefore the truncated series solution obtained from LAPM is

$$
\begin{aligned}
y(t)= & y_{0}+y_{1}+y_{2}+\cdots, \\
y(t)= & \frac{t^{\alpha}}{\Gamma(1+\alpha)}+\frac{2 t^{2 \alpha}}{\Gamma(1+2 \alpha)}+\frac{4 t^{3 \alpha}}{\Gamma(1+3 \alpha)}-\frac{t^{3 \alpha} \Gamma(1+2 \alpha)}{(\Gamma(1+\alpha))^{2} \Gamma(1+3 \alpha)} \\
& +\frac{8 t^{4 \alpha}}{\Gamma(1+4 \alpha)}-\frac{2 t^{4 \alpha} \Gamma(1+2 \alpha)}{(\Gamma(1+\alpha))^{2} \Gamma(1+4 \alpha)}-\cdots .
\end{aligned}
$$

The aim is to study the mathematical behavior of the result as the order of the fractional derivative changes. It was formally shown by Khan et al. [21] that this goal can be achieved by forming Padé approximants [22] which have the advantage of manipulating the polynomial approximation into a rational function to gain more information about $y(t)$. To consider the behavior of a solution of different values of $\alpha$, we will take advantage of Eq. (40) available for $0<\alpha \leq 1$ and consider the following three special cases.

Case I: Setting $\alpha=1$ in Eq. (40), we reproduce the approximate solution obtained in Eq. (40), given by the Taylor expansion of $y(t)$ at $t=0$ of the LAPM solution, as follows:

$$
y(t)=t+t^{2}+0.333333 t^{3}-0.333333 t^{4}-0.466667 t^{5}+O\left(t^{6}\right)
$$

The Taylor expansion of $y(t)$ at $t=0$ of the exact solution (25) is

$$
y(t)=0.999999 t+0.999999 t^{2}+0.333333 t^{3}-0.333333 t^{4}-0.466667 t^{5}+O\left(t^{6}\right)
$$

It indicates that both the Taylor expansions at $t=0$ of the LAPM solution and the exact solution coincide very well. In order to improve the LAPM solution, the Padé approximant 
is introduced. It is known that there exists the $\left[\frac{L}{M}\right]$ Padé approximant which satisfies

$$
\sum_{n=0}^{\infty} y_{n}=\frac{P_{L}(t)}{Q_{M}(t)}-O\left(t^{L+M+1}\right)=\left[\frac{L}{M}\right]+O\left(t^{31}\right)
$$

By using Mathematica, the $\left[\frac{15}{15}\right]$ Padé approximant gives that the rational approximation obtained from the solution in Eq. (42) is determined to be

$$
\left[\frac{15}{15}\right]=\frac{t+0.324979 t^{2}-0.225729 t^{3}-0.443244 t^{4} \cdots+0.000544953 t^{15}}{1-0.675021 t+0.115958 t^{2}-0.000861535 t^{3}+\cdots-1.27233 \times 10^{-9} t^{15}} .
$$

Figures 1-2 represent the comparisons between the exact solution, the LAM and the LAPM solutions in problem 1 . They show that the LAM solutions diverge rapidly after

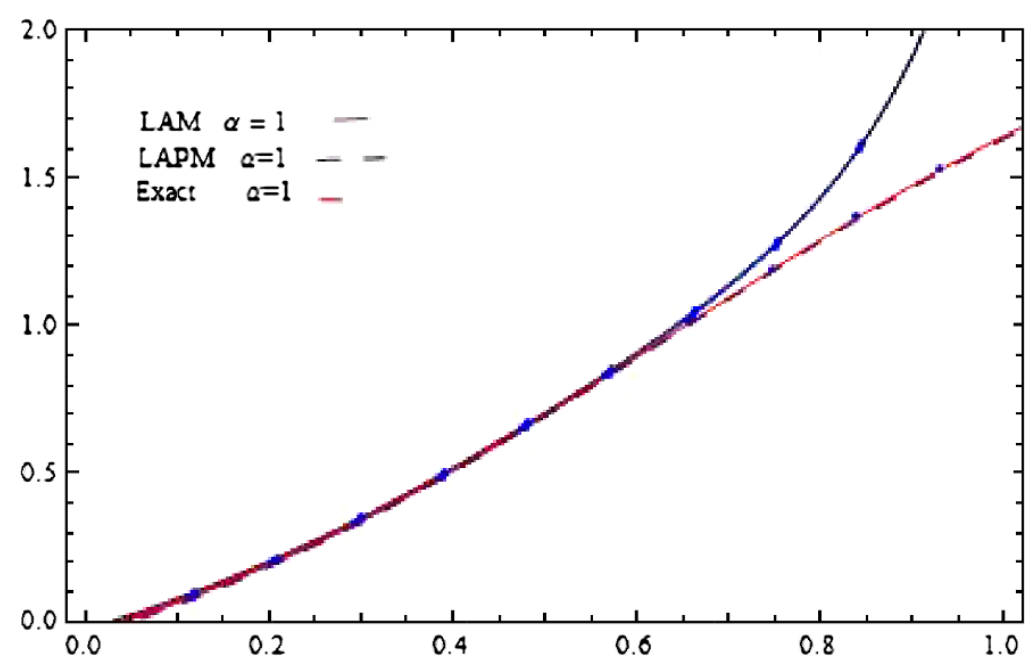

Figure 1 The approximate solutions solved by different methods in problem 1 for $\alpha=1$.

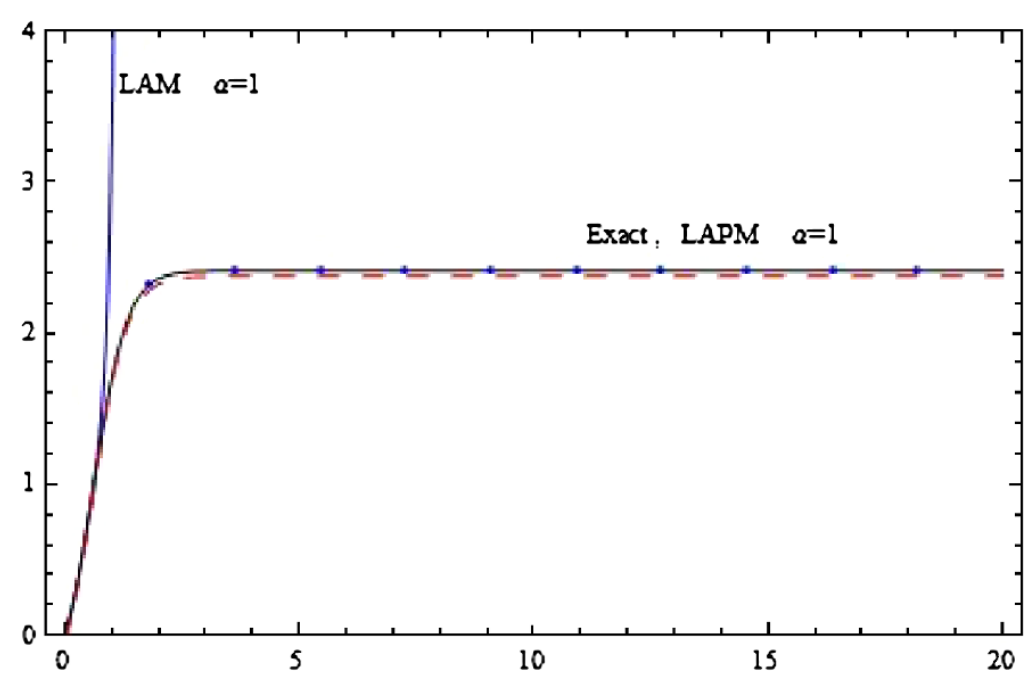

Figure 2 The approximate solutions solved by different methods in problem 1 for $\alpha=1$. 
Table 1 Numerical results of the Riccati equation in problem 1

\begin{tabular}{lllll}
\hline $\boldsymbol{t}$ & $\begin{array}{l}\text { GEM } \boldsymbol{\alpha}=\mathbf{1} \\
\boldsymbol{y}(\boldsymbol{t})\end{array}$ & $\begin{array}{l}\text { LAPM } \boldsymbol{\alpha}=\mathbf{1} \\
\boldsymbol{y}(\boldsymbol{t})\end{array}$ & $\begin{array}{l}\text { Exact solution } \\
\boldsymbol{y ( t )}\end{array}$ & Absolute error \\
\hline 0.1 & 0.1000000000 & 0.1102952044 & 0.1102951969 & $7.5 \times 10^{-9}$ \\
0.2 & 0.2419000000 & 0.2419783394 & 0.2419767996 & $1.5 \times 10^{-6}$ \\
0.3 & 0.3580039000 & 0.3951442714 & 0.3951048487 & 0.00003942275 \\
0.4 & 0.5167880007 & 0.5682377001 & 0.5678121663 & 0.00042553377 \\
0.5 & 0.6934386 & 0.7588607194 & 0.7580143934 & 0.00084632599 \\
\hline
\end{tabular}

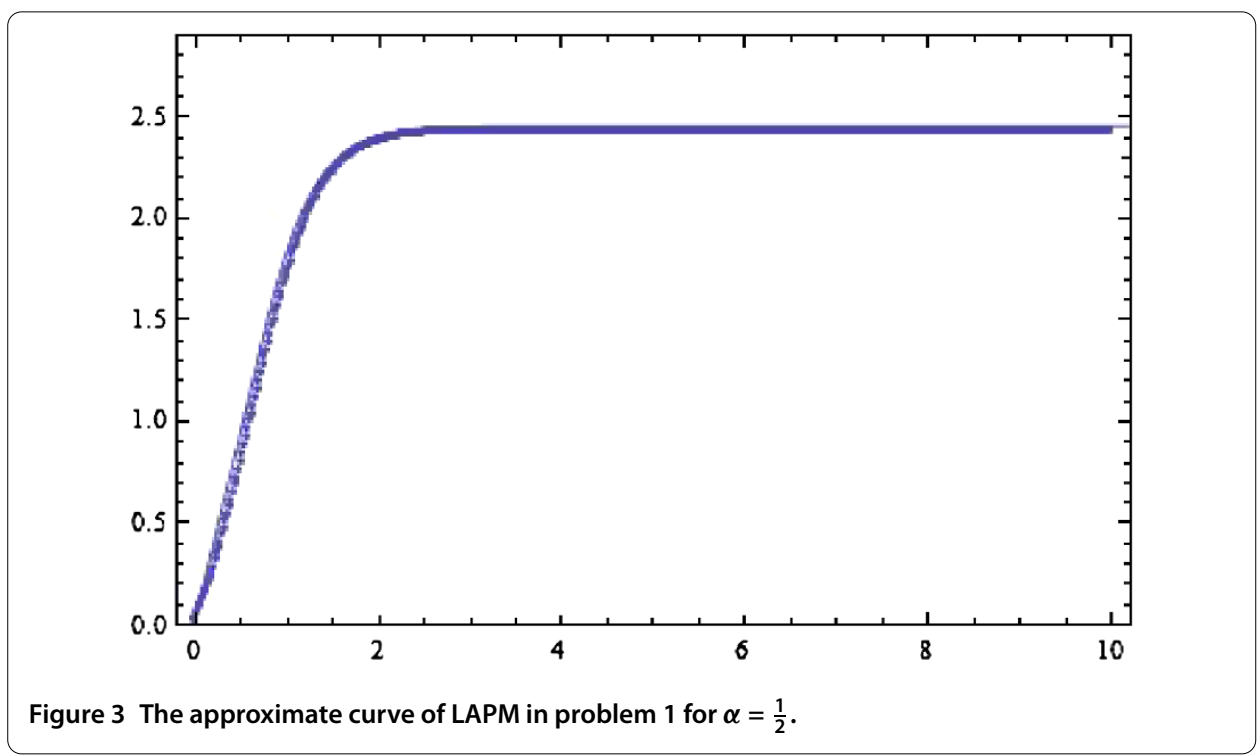

$t=1$. However, they represent that the LAPM solution demonstrates a good convergence through the applicable domain. Table 1 shows the absolute errors of the LAPM solution in comparison with the exact and GEM solutions in problem 1.

Case II: Let us examine the case $\alpha=\frac{1}{2}$, the approximate solution obtained in Eq. (40) given by the Taylor expansion of $y(t)$ at $t=0$ has reproduced as

$$
y(t)=1.12938 \sqrt{t}+2 t+2.05121 t^{\frac{3}{2}}-0.27324 t^{\frac{5}{2}} \cdots .
$$

For simplicity, let $t^{\frac{1}{2}}=z$, then

$$
y(z)=1.12938 z+2 z^{2}+2.05121 z^{3}-\cdots .
$$

Calculating the $\left[\frac{28}{28}\right]$ Padé approximation and recalling that $z=t^{\frac{1}{2}}$, we obtain

$$
\left[\frac{28}{28}\right]=\frac{1.12838 t^{1 / 2}+0.298577 t^{3 / 2}-0.455749 t^{5 / 2} \cdots+25.3824 t^{27 / 2}}{1-0.65902 t-0.0790958 t^{2}-0.256754 t^{3} \cdots 0.00733938 t^{14}}
$$

Figure 3 represents the LAPM solution in problem 1 for $\alpha=\frac{1}{2}$.

Case III: Here, taking $\alpha=\frac{3}{4}$ in Eq. (40), the approximate solution has been replicated by

$$
y(t)=1.08807 t^{\frac{3}{4}}+1.50451 t^{\frac{7}{4}}-0.617351 t^{\frac{9}{4}} \cdots 6.83286 \times 10^{-11} t^{\frac{119}{4}} .
$$




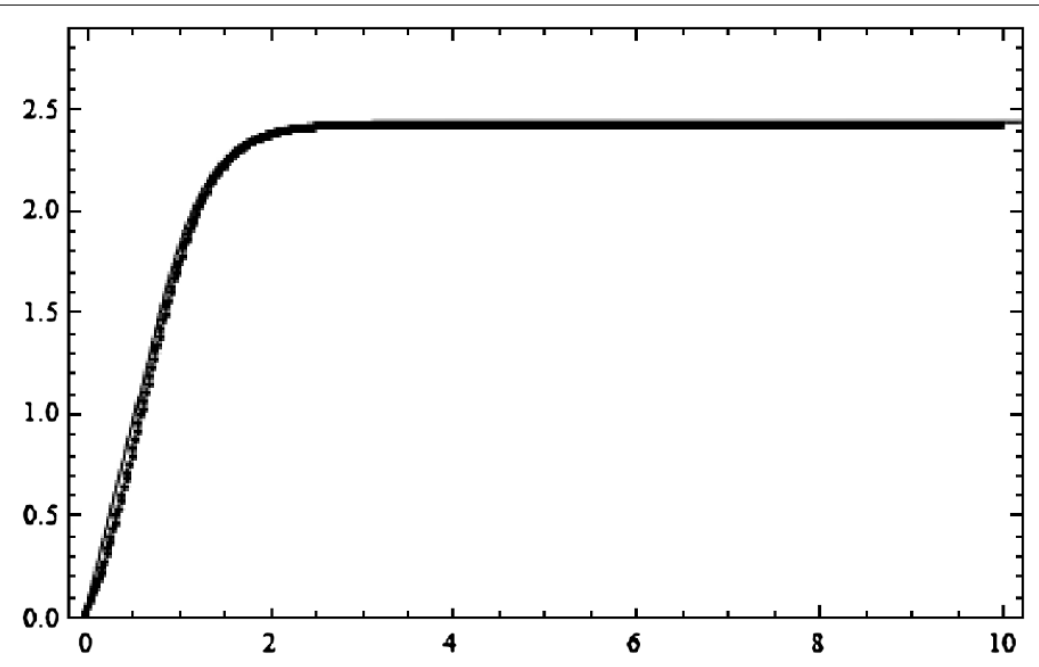

Figure 4 The approximate curve of LAPM in problem 1 for $\alpha=\frac{3}{4}$.

Table 2 Numerical results of the Riccati equation in problem 1 for $\alpha=1, \frac{1}{2}, \frac{3}{4}$

\begin{tabular}{llllll}
\hline $\boldsymbol{t}$ & $\boldsymbol{\alpha}=\frac{\mathbf{1}}{\mathbf{2}}$ GEM & $\boldsymbol{\alpha}=\frac{\mathbf{1}}{\mathbf{2}}$ LAPM & $\boldsymbol{\alpha}=\frac{\mathbf{3}}{\mathbf{4}}$ GEM & $\boldsymbol{\alpha}=\frac{\mathbf{3}}{\mathbf{4}}$ LAPM & $\boldsymbol{\alpha}=\mathbf{1}$ LAPM \\
\hline 0.1 & 0.3568251903 & 0.3568031433 & 0.1934884034 & 0.1934012434 & 0.1102952044 \\
0.2 & 0.9228652311 & 0.9228654512 & 0.4546091238 & 0.4546025138 & 0.2419783394 \\
0.3 & 1.6341391963 & 1.6341391234 & 0.7840321022 & 0.7840324522 & 0.3951442714 \\
0.4 & 2.2044414876 & 2.2044414576 & 1.1619801122 & 1.1619856232 & 0.5682377001 \\
0.5 & 2.4004512311 & 2.4004476111 & 1.5438814841 & 1.5438814521 & 0.7588607194 \\
0.6 & 2.0414276521 & 2.0414345521 & 1.8736212813 & 1.873658343 & 0.8840411201 \\
0.7 & 2.4142176521 & 2.414888821 & 2.1129313512 & 2.112943562 & 1.0827124311 \\
0.8 & 2.4142456641 & 2.4142478941 & 2.2602500123 & 2.260134223 & 1.2820124311 \\
0.9 & 2.4142456047 & 2.4142455667 & 2.339920199 & 2.339134229 & 1.4740612089 \\
1 & 2.4142410607 & 2.4142312137 & 2.3795146712 & 2.37935612 & 1.6515902374 \\
\hline
\end{tabular}

For simplicity, let $t^{\frac{1}{4}}=z$; then

$$
y(t)=1.08807 z^{3}+1.50451 z^{7}-0.617351 z^{9} \cdots 6.83286 \times 10^{-11} z^{119} .
$$

Calculating the $\left[\frac{59}{59}\right]$ Padé approximants and recalling that $z=t^{\frac{1}{4}}$, we achieve

$$
\left[\frac{59}{59}\right]=\frac{1.08807 t^{3 / 4}-0.173349 t^{5 / 4}+0.881229 t^{7 / 4} \cdots-0.78027 t^{58 / 4}}{1-0.159319 \sqrt{t}-0.57283 t+0.138625 t^{3 / 2} \cdots-16246 \times 10^{-8} t^{29 / 2}}
$$

Figure 4 shows the LAPM solution in problem 1 for $\alpha=\frac{3}{4}$.

Table 2 shows the results of the fractional Riccati equation in test problem 1 of the LAPM approximant solution in comparison with the different values of $\alpha=1, \frac{1}{2}, \frac{3}{4}$. The technique described above was translated into a Mathematica program and run on a Pentium-4 PC to investigate the effects of various values of $\alpha=1, \frac{1}{2}, \frac{3}{4}$ on the fractional Riccati differential equation. The graphical results are in good agreement with the results of the exact solution.

Test problem 2. Consider the nonlinear Riccati differential equation

$$
D_{t}^{\alpha} y(t)=1-y^{2}(t)
$$


with the initial condition

$$
y(0)=0 \text {. }
$$

The exact solution [9] was found to be

$$
y(t)=\frac{e^{2 t}-1}{e^{2 t}+1} .
$$

First, applying the Laplace transform to both sides of Eq. (51), we get

$$
\mathcal{L}\left[D_{t}^{\alpha}(y)\right]=\mathcal{L}[1]-\mathcal{L}\left[y^{2}\right]
$$

Using the property of the Laplace transform yields

$$
s^{\alpha} \mathcal{L}[y]-y(0)=\frac{1}{s}-\mathcal{L}\left[y^{2}\right]
$$

Utilizing the initial conditions from Eq. (52), it becomes

$$
s^{\alpha} \mathcal{L}[y]=\frac{1}{s}-\mathcal{L}\left[y^{2}\right]
$$

or

$$
\mathcal{L}[y]=\frac{1}{s^{\alpha+1}}-\frac{1}{s^{\alpha}} \mathcal{L}\left[y^{2}\right]
$$

Substituting Eqs. (15) and (16) into Eq. (57), the result is

$$
\mathcal{L}\left[\sum_{n=0}^{\infty} y_{n}\right]=\left(\frac{1}{s^{\alpha+1}}\right)-\left(\frac{1}{s^{\alpha}}\right) \mathcal{L}\left[P(t) \sum_{n=0}^{\infty} A_{n}\right] .
$$

Matching both sides of Eq. (58) yields the following iterative algorithm:

$$
\begin{aligned}
& \mathcal{L}\left[y_{0}\right]=\frac{1}{s^{\alpha+1}}, \\
& \mathcal{L}\left[y_{1}\right]=-\frac{1}{s^{\alpha}} \mathcal{L}\left[A_{0}\right], \\
& \mathcal{L}\left[y_{2}\right]=-\frac{1}{s^{\alpha}} \mathcal{L}\left[A_{1}\right], \\
& \vdots \\
& \mathcal{L}\left[y_{n}\right]=-\frac{1}{s^{\alpha}} \mathcal{L}\left[A_{n-1}\right] \quad(n \geq 1) .
\end{aligned}
$$

Applying the inverse fractional Laplace transform to Eq. (59), hence the value $y_{0}$ is

$$
y_{0}=\frac{t^{\alpha}}{\Gamma(\alpha+1)} \text {. }
$$


Substituting the value of $y_{0}$ in Eq. (60), the first Adomian polynomial $A_{0}$ is obtained, then substituting $y_{0}$ and $A_{0}$ in Eq. (60) and proceeding in a similar way, the other terms $y_{2}, y_{3}, y_{4}, \ldots$ can be computed recursively. The first twelve components of the solution are

$$
\begin{aligned}
y_{1}= & -\frac{t^{3 \alpha} \Gamma(1+2 \alpha)}{(\Gamma(1+\alpha))^{2} \Gamma(1+2 \alpha)}, \\
y_{2}= & \frac{2 t^{5 \alpha} \Gamma(1+2 \alpha) \Gamma(1+4 \alpha)}{(\Gamma(1+2 \alpha))^{3} \Gamma(1+3 \alpha) \Gamma(1+5 \alpha)}, \\
y_{3}= & -\frac{t^{7 \alpha}(\Gamma(1+2 \alpha))^{2} \Gamma(1+6 \alpha)}{(\Gamma(1+\alpha))^{4}(\Gamma(1+3 \alpha))^{2} \Gamma(1+7 \alpha)} \\
& -\frac{t^{7 \alpha} \Gamma(1+2 \alpha) \Gamma(1+4 \alpha) \Gamma(1+6 \alpha)}{(\Gamma(1+\alpha))^{4}(\Gamma(1+3 \alpha))^{2} \Gamma(1+5 \alpha) \Gamma(1+7 \alpha)}, \\
\vdots & \\
y_{12}= & \frac{64 t^{25 \alpha}(\Gamma(1+2 \alpha))^{4}(\Gamma(1+4 \alpha))^{4}(\Gamma(1+2 \alpha))^{2} \Gamma(1+12 \alpha) \Gamma(1+24 \alpha)}{(\Gamma(1+\alpha))^{13}(\Gamma(1+3 \alpha))^{4}(\Gamma(1+5 \alpha))^{4}(\Gamma(1+11 \alpha))^{2} \Gamma(1+13 \alpha) \Gamma(1+25 \alpha)} \\
& +\frac{64 t^{25 \alpha}(\Gamma(1+2 \alpha))^{6}(\Gamma(1+6 \alpha))^{2}(\Gamma(1+10 \alpha))^{2} \Gamma(1+12 \alpha) \Gamma(1+24 \alpha)}{(\Gamma(1+\alpha))^{13}(\Gamma(1+3 \alpha))^{6}(\Gamma(1+7 \alpha))^{4}(\Gamma(1+11 \alpha))^{2} \Gamma(1+13 \alpha) \Gamma(1+25 \alpha)} .
\end{aligned}
$$

Therefore the truncated series solution is obtained as

$$
\begin{aligned}
y(t)= & y_{0}+y_{1}+y_{2}+\cdots+y_{12} \\
y(t)= & \frac{1}{\Gamma(1+\alpha)}-\frac{t^{3 \alpha} \Gamma(1+3 \alpha)}{(\Gamma(1+\alpha))^{2} \Gamma(1+3 \alpha)}+\frac{2 t^{5 \alpha} \Gamma(1+2 \alpha) \Gamma(1+4 \alpha)}{(\Gamma(1+\alpha))^{3} \Gamma(1+3 \alpha) \Gamma(1+5 \alpha)} \\
& -\frac{t^{7 \alpha}(\Gamma(1+2 \alpha))^{2} \Gamma(1+6 \alpha)}{(\Gamma(1+\alpha))^{4}(\Gamma(1+3 \alpha))^{2} \Gamma(1+7 \alpha)} \\
& -\frac{4 t^{7 \alpha} \Gamma(1+2 \alpha) \Gamma(1+4 \alpha) \Gamma(1+6 \alpha)}{(\Gamma(1+\alpha))^{4}(\Gamma(1+3 \alpha))^{2} \Gamma(1+5 \alpha) \Gamma(1+7 \alpha)}-\cdots
\end{aligned}
$$

The plan is to study the mathematical performance of the solution of LAPM as the order of the fractional derivative changes. To consider the behavior of a solution of different values of $\alpha$, we will take advantage of the explicit formula Eq. (69) available for $0<\alpha \leq 1$ and consider the following three special cases.

Case I: Setting $\alpha=1$ in Eq. (69), we reproduce the approximate solution obtained in Eq. (69) given by the Taylor expansion of $y(t)$ at $t=0$ of the LAPM solution as follows:

$$
\begin{aligned}
y(t)= & t-\frac{1}{3} t^{3}+\frac{2}{15} t^{5}-\frac{17}{315} t^{7}+\frac{62}{2,835} t^{9}-\frac{1,382}{155,925} t^{11}+\frac{21,844}{6,081,075} t^{13} \\
& -\frac{929,569}{638,512,875} t^{15}+\frac{6,404,582}{10,854,718,875} t^{17}-\frac{443,861,162}{1,856,156,927,625} t^{19} \\
& -\frac{18,888,466,084}{194,896,477,400,625} t^{21}+\frac{113,927,491,862}{2,900,518,163,668,125} t^{23} \\
& -\frac{58,870,668,456,604}{3,698,160,658,676,859,375} t^{25}
\end{aligned}
$$




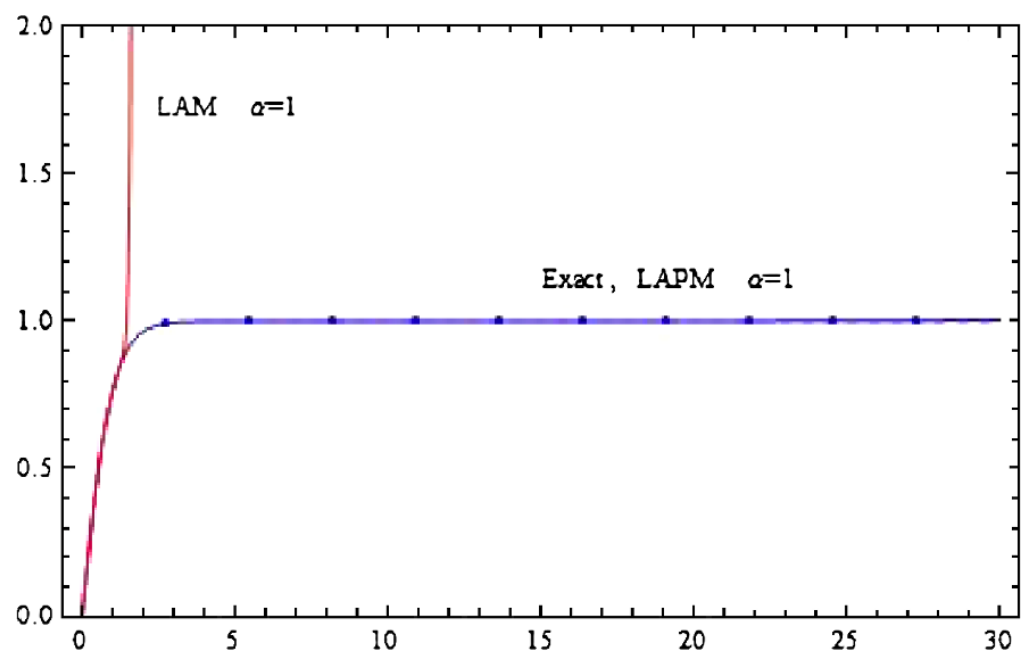

Figure 5 The approximate solutions solved by different methods in problem 2 for $\alpha=1$.

It is known that there exists the $\left[\frac{L}{M}\right]$ Padé approximant which satisfies

$$
\sum_{n=0}^{\infty} y_{n}=\frac{P_{L}(t)}{Q_{M}(t)}-O\left(t^{L+M+1}\right)=\left[\frac{L}{M}\right]+O\left(t^{31}\right) .
$$

By using Mathematica, the Padé approximation gives that the truncated series obtained from the LAPM solution in Eq. (70) is determined to be

$$
\left[\frac{13}{12}\right]=\frac{t+\frac{11}{75} t^{3}+\frac{3}{575} t^{5}+\frac{4}{60,375} t^{7}+\frac{2}{6,194,475} t^{9}+\frac{1}{1,930,611,375} t^{11}+\frac{1}{7,905,853,580,625} t^{13}}{1+\frac{12}{25} t^{2}+\frac{11}{345} t^{4}+\frac{8}{12,075} t^{6}+\frac{2}{382,375} t^{8}+\frac{8}{526,530,375} t^{10}+\frac{1}{86,877,511,875} t^{12}} .
$$

From Figure 5, the presented result is in a good agreement with the exact result for $\alpha=1$. Figure 5 represents the comparisons between the exact solution, the LAM, and the LAPM solutions for problem 2 . It shows that the LAM solutions diverge rapidly after $t=2$. However, it represents that the LAPM solution demonstrates a good convergence through the applicable domain. Table 3 shows the absolute errors of the LAPM solution in comparison with the exact solution.

Case II: Here we examine the case $\alpha=\frac{1}{2}$ in Eq. (69), we replicate the approximate solution obtained in Eq. (69) given by

$$
y(t)=1.12838 \sqrt{t}-0.957798 t^{\frac{3}{2}}+1.3008 t^{\frac{5}{2}} \cdots+249.383 t^{\frac{25}{2}} .
$$

For simplicity, let $t^{\frac{1}{2}}=z$; then

$$
y(t)=1.12838 z-0.957798 z^{3}+1.3008 z^{5} \cdots+249.383 z^{25} .
$$

Calculating the $\left[\frac{13}{12}\right]$ Padé approximation and recalling that $z=t^{\frac{1}{2}}$, we get

$$
\left[\frac{13}{12}\right]=\frac{1.12838 t^{1 / 2}+5.75063 t^{3 / 2}-10.9207 t^{5 / 2} \cdots+0.0116803 t^{13 / 2}}{1+5.9519 t+13.5718 t^{2}-0.256754 t^{3} \cdots+0.113742 t^{6}} .
$$


Table 3 Comparison results of the Riccati equation in problem 2 for $\alpha=1$

\begin{tabular}{rlll}
\hline $\boldsymbol{t}$ & $\begin{array}{l}\text { LAPM } \boldsymbol{\alpha}=\mathbf{1} \\
\boldsymbol{y}(\boldsymbol{t})\end{array}$ & $\begin{array}{l}\text { Exact solution } \\
\boldsymbol{y}(\boldsymbol{t})\end{array}$ & Absolute error \\
\hline 1.0 & 0.7615941560 & 0.7615941560 & $0.01235728510 \times 10^{-13}$ \\
2.0 & 0.9640275801 & 0.9640275801 & $0.00524212251 \times 10^{-12}$ \\
3.0 & 0.9950547537 & 0.9950547537 & $0.00816114713 \times 10^{-12}$ \\
4.0 & 0.9993292997 & 0.9993292997 & $1.15746178216 \times 10^{-12}$ \\
5.0 & 0.9999092043 & 0.9999092043 & $6.17826138530 \times 10^{-11}$ \\
6.0 & 0.9999877117 & 0.9999877117 & $4.55279023510 \times 10^{-11}$ \\
7.0 & 0.9999983377 & 0.9999983369 & $7.57246128510 \times 10^{-10}$ \\
8.0 & 0.9999997813 & 0.9999997749 & $6.33977368904 \times 10^{-9}$ \\
9.0 & 1.0000000063 & 0.9999999695 & $3.67962922016 \times 10^{-8}$ \\
10.0 & 1.0000001574 & 0.9999999958 & $1.61485463823 \times 10^{-7}$ \\
15.0 & 1.000000000 & 1.0000000000 & 0.00002076502676 \\
20.0 & 1.0000000000 & 1.0000000000 & 0.00030117782151 \\
25.0 & 1.0000000000 & 1.0000000000 & 0.00161702848408 \\
30.0 & 1.0000000000 & 1.0000000000 & 0.00510751644687 \\
\hline
\end{tabular}

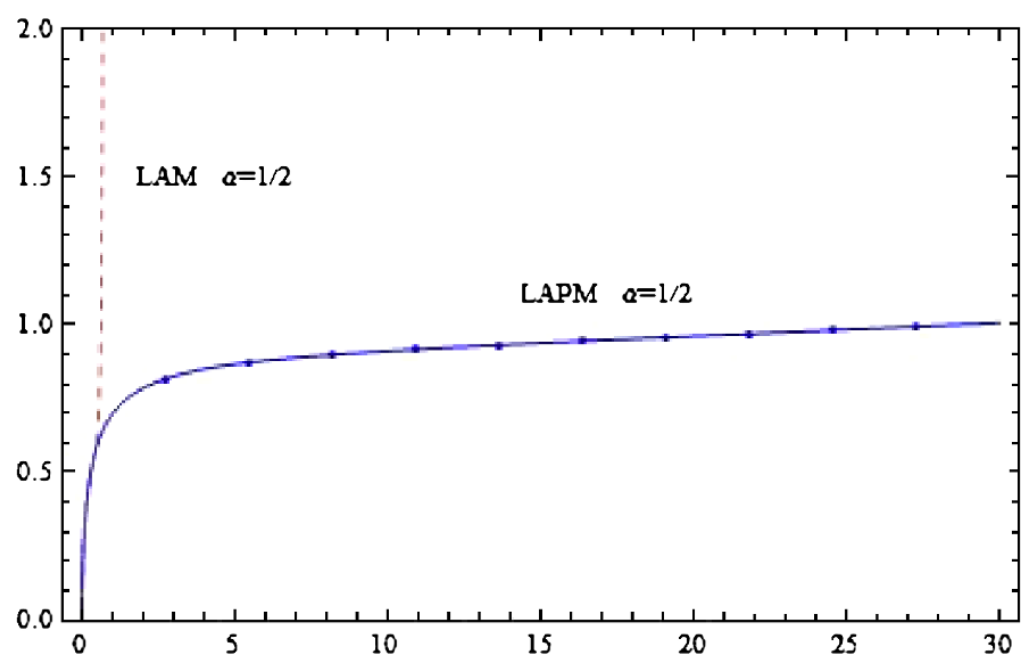

Figure 6 The approximate solutions solved by different methods in problem 2 for $\alpha=\frac{1}{2}$.

Figure 6 shows the $\left[\frac{13}{12}\right]$ Pade approximants of $y(t)$ in LAM and LAPM for $\alpha=\frac{1}{2}$. Figure 6 illustrates the comparisons between the LAM solution and the LAPM solution in problem 2 for $\alpha=\frac{1}{2}$.

Case III: In this case we examine the LAPM when $\alpha=\frac{3}{4}$ in Eq. (69)

$$
y(t)=1.08807 t^{\frac{3}{4}}-0.617351 t^{\frac{9}{4}}+0.485984 t^{\frac{15}{4}} \cdots+0.13387 t^{\frac{75}{4}} .
$$

For simplicity, let $t^{\frac{3}{4}}=z$; then

$$
y(t)=1.08807 z^{3}-0.617351 z^{9}+0.485984 z^{15} \cdots+0.13387 z^{75} .
$$




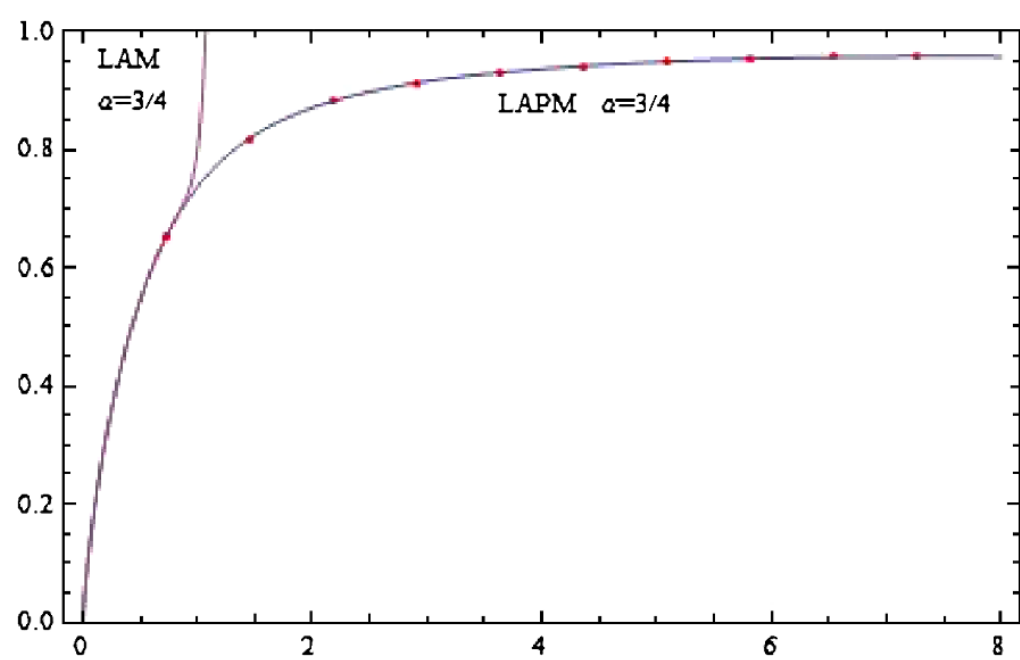

Figure 7 The approximate curves of problem 2 for $\alpha=\frac{3}{4}$.

Table 4 Numerical results of the Riccati equation of problem 2 for $\alpha=1, \frac{1}{2}, \frac{3}{4}$

\begin{tabular}{rlllll}
\hline $\boldsymbol{t}$ & $\boldsymbol{\alpha}=\frac{\mathbf{1}}{\mathbf{2}}$ GEM & $\boldsymbol{\alpha}=\frac{\mathbf{1}}{\mathbf{2}}$ LAPM & $\boldsymbol{\alpha}=\frac{\mathbf{3}}{\mathbf{4}}$ GEM & $\boldsymbol{\alpha}=\frac{\mathbf{3}}{\mathbf{4}}$ LAPM & $\boldsymbol{\alpha}=\mathbf{1}$ LAPM \\
\hline 1 & 1.1283791670 & 0.69873925716 & 1.08806525252 & 0.73683666979 & 0.7615941560 \\
2 & 0.8200613571 & 0.78565566383 & 0.88785292084 & 0.87018299629 & 0.9640275801 \\
3 & 1.1896048240 & 0.82585713776 & 1.11809163866 & 0.91495001137 & 0.9950547537 \\
4 & 0.7211473382 & 0.85006608584 & 0.84593506046 & 0.93590393958 & 0.9993292997 \\
5 & 1.2627089888 & 0.86673312218 & 1.15537415211 & 0.94734255879 & 0.9999092043 \\
6 & 0.5919620577 & 0.87923035124 & 0.79099259853 & 0.95361548723 & 0.9999877117 \\
7 & 1.3249356376 & 0.88919590450 & 1.19828883587 & 0.95638359853 & 0.9999983377 \\
8 & 0.4724965813 & 0.89752861875 & 0.72400539873 & 0.95643868318 & 0.9999997813 \\
9 & 1.3489616923 & 0.90476369883 & 1.24172445342 & 0.95425989627 & 1.0000000063 \\
10 & 0.4240319436 & 0.91123881947 & 0.65212406998 & 0.95020154024 & 1.0000001574 \\
\hline
\end{tabular}

Calculating the $\left[\frac{37}{37}\right]$ Padé approximation and recalling that $z=t^{\frac{3}{4}}$, we get

$$
\begin{aligned}
{\left[\frac{37}{37}\right]=} & \left(1.08807 t^{3 / 4}+9.60386 \times 10^{-10} t-8.67419 \times 10^{-9} t^{5 / 4} \ldots\right. \\
& \left.-4.42862 \times 10^{-12} t^{37 / 4}\right) \\
& /\left(1+8.82655 \times 10^{-10} t^{1 / 4}-7.97212 \times 10^{-9} \sqrt{t} \ldots\right. \\
& \left.-2.87725 \times 10^{-12} t^{37 / 4}\right) .
\end{aligned}
$$

Figure 7 shows the $\left[\frac{37}{37}\right]$ Padé approximants of $y(t)$ in LAPM for $\alpha=\frac{3}{4}$. Table 4 shows the comparison results of the fractional Riccati equation in test problem 2 of the LAPM solution in comparison with the different values of $\alpha=1, \frac{1}{2}, \frac{3}{4}$. The procedure described above was translated into Mathematica program and run on a Pentium-4 PC to investigate the effects of special values of $\alpha=1, \frac{1}{2}, \frac{3}{4}$ for the fractional Riccati differential equation. $y(t)$ is evaluated up to $n=12$ and plotted in Figure 8 .

\section{Conclusions}

Most of the real physical problems can be best modeled with fractional differential equations. Besides modeling, the solution techniques and their reliabilities are most important 


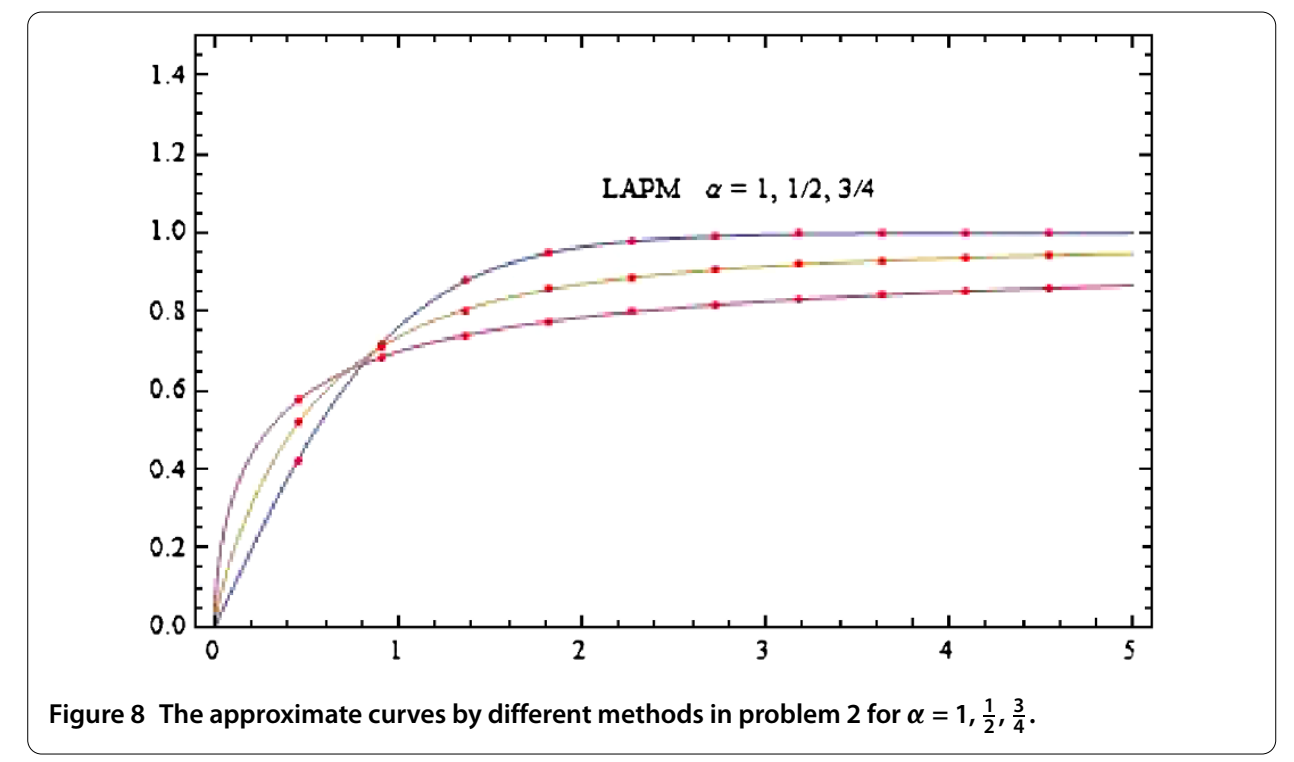

to catch critical points at which a sudden divergence or bifurcation starts. Therefore, high accuracy solutions are always needed. Here, we have implemented the Adomian decomposition method coupled with the Laplace transformation and the Padé approximation on the Ricatti differential equation with fractional order. From the test problems considered here, it can be easily seen that LAPM obtains results as accurate as possible. Thus, it can be concluded that the LAPM methodology is very dominant and efficient in finding approximate solutions, and comparison has been made with GEM. This paper can be used as a standard paradigm for other applications. The results of LAPM have been compared with exact solutions and ref. [16] for $\alpha=1$.

\section{Competing interests}

The authors declare that they have no competing interests.

Authors' contributions

The authors have equal contributions and they have approved the final version of the manuscript.

\section{Author details}

'Department of Mathematical Sciences, University of Karachi, Karachi, 75270, Pakistan. ${ }^{2}$ Department of Mathematical Sciences, Federal Urdu University Arts, Science and Technology, Karachi, 75300, Pakistan.

\section{Acknowledgements}

The authors would like to express their sincere gratitude to the referees for their careful assessment and suggestions regarding the initial version of the manuscript. The author Najeeb Alam Khan is highly thankful and grateful to the Dean of Faculty of Sciences, University of Karachi, Karachi-75270, Pakistan for facilitating this research work.

\section{Received: 8 January 2013 Accepted: 29 May 2013 Published: 26 June 2013}

\section{References}

1. Podlubny, I: Fractional Differential Equations. Academic Press, New York (1999)

2. Khan, NA, Jamil, M, Ara, A, Das, S: Explicit solution of time-fractional batch reactor system. Int. J. Chem. React. Eng. 9, Article ID A91 (2011)

3. Feliu-Batlle, V, Perez, R, Rodriguez, L: Fractional robust control of main irrigation canals with variable dynamic parameters. Control Eng. Pract. 15, 673-686 (2007)

4. Podlubny, I: Fractional-order systems and controllers. IEEE Trans. Autom. Control 44(1), 208-214 (1999)

5. Garrappa, R: On some explicit Adams multistep methods for fractional differential equations. J. Comput. Appl. Math. 229, 392-399 (2009)

6. Jamil, M, Khan, NA: Slip effects on fractional viscoelastic fluids. Int. J. Differ. Equ. 2011, Article ID 193813 (2011)

7. Abbasbandy, S: Homotopy perturbation method for quadratic Riccati differential equation and comparison with Adomian's decomposition method. Appl. Math. Comput. 172(1), 485-490 (2006) 
8. Odibat, Z, Momani, S: Modified homotopy perturbation method: application to quadratic Riccati differential equation of fractional order. Chaos Solitons Fractals 36(1), 167-174 (2008)

9. Khan, NA, Ara, A, Jamil, M: An efficient approach for solving the Riccati equation with fractional orders. Comput. Math. Appl. 61, 2683-2689 (2011)

10. Aminkhah, $\mathrm{H}$, Hemmatnezhad, M: An efficient method for quadratic Riccati differential equation. Commun. Nonlinear Sci. Numer. Simul. 15, 835-839 (2010)

11. Abbasbandy, S: Iterated He's homotopy perturbation method for quadratic Riccati differential equation. Appl. Math. Comput. 175, 581-589 (2006)

12. Cang, J, Tan, Y, Xu, H, Liao, SJ: Series solutions of non-linear Riccati differential equations with fractional order. Chaos Solitons Fractals 40, 1-9 (2009)

13. Tan, Y, Abbasbandy, S: Homotopy analysis method for quadratic Riccati differential equation. Commun. Nonlinear Sci. Numer. Simul. 13, 539-546 (2008)

14. Gülsu, M, Sezer, M: On the solution of the Riccati equation by the Taylor matrix method. Appl. Math. Comput. 176(2), 414-421 (2006)

15. Li, Y, Hu, L: Solving fractional Riccati differential equations. In: Third International Conference on Information and Computing Using Haar Wavelet. IEEE (2010). doi:10.1109/ICIC.2010.86

16. Tsai, $\mathrm{P}, \mathrm{Chen}, \mathrm{CK}$ : An approximate analytic solution of the nonlinear Riccati differential equation. J. Franklin Inst. 347 , 1850-1862 (2010)

17. Zeng, DQ, Qin, YM: The Laplace-Adomian-Pade technique for the seepage flows with the Riemann-Liouville derivatives. Commun. Frac. Calc. 3, 26-29 (2012)

18. Khan, Y, Diblik, J, Faraz, N, Smarda, Z: An efficient new perturbative Laplace method for space-time fractional telegraph equations. Adv. Differ. Equ. 2012, Article ID 204 (2012)

19. Odibat, Z, Momani, S: An algorithm for the numerical solution of differential equations of fractional order. J. Appl. Math. Inform. 26, 15-27 (2008)

20. Agarwal, RP: A propos d'une note de M. Pierre Humbert. C. R. Acad. Sci. Paris 236(21), 2031-2032 (1953)

21. Khan, NA, Jamil, M, Ara, A, Khan, NU: On efficient method for system of fractional differential equations. Adv. Differ. Equ. 2011, Article ID 303472 (2011)

22. Baker, GA: Essentials of Padé Approximants. Academic Press, London (1975)

doi:10.1186/1687-1847-2013-185

Cite this article as: Khan et al.: Fractional-order Riccati differential equation: Analytical approximation and numerical results. Advances in Difference Equations 2013 2013:185.

\section{Submit your manuscript to a SpringerOpen ${ }^{\circ}$ journal and benefit from:}

- Convenient online submission

- Rigorous peer review

- Immediate publication on acceptance

- Open access: articles freely available online

- High visibility within the field

- Retaining the copyright to your article 